\title{
Proteogenomic characterization and integrative analysis of glioblastoma multiforme
}

\author{
Ying-Chun Song ${ }^{1, *}$, Gai-Xia Lu, ${ }^{1, *}$, Hong-Wei Zhang ${ }^{2, *}$, Xiao-Ming Zhong ${ }^{3, *}$, Xian- \\ Ling Cong ${ }^{4, *}$, Shao-Bo Xue ${ }^{1, *}$, Rui Kong ${ }^{1, *}$, Dan Li $^{1}$, Zheng-Yan Chang ${ }^{5}$, Xiao-Feng \\ Wang ${ }^{6}$, Yun-Jie Zhang ${ }^{6}$, Ran Sun ${ }^{4}$, Li Chai ${ }^{1}$, Ru-Ting Xie ${ }^{5}$, Ming-Xiang Cai ${ }^{1}$, Ming \\ Sun ${ }^{1}$, Wei-Qing Mao', Hui-Qiong Yang ${ }^{5}$, Yun-Chao Shao ${ }^{6}$, Su-Yun Fan ${ }^{1}$, Ting-Miao

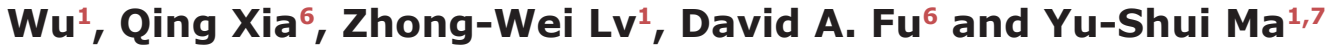 \\ ${ }^{1}$ Department of Nuclear Medicine, Shanghai Tenth People's Hospital, Tongji University School of Medicine, Shanghai 200072, \\ China \\ ${ }^{2}$ Institute of Health Sciences, Shanghai Institutes for Biological Sciences, Chinese Academy of Sciences, Shanghai 200025, \\ China \\ ${ }^{3}$ Department of Radiology, Jiangxi Provincial Tumor Hospital/Ganzhou City People's Hospital, Nanchang 330029, China \\ ${ }^{4}$ Department of Biobank, China-Japan Unoin Hospital, Jilin University, Changchun 130033, China \\ ${ }^{5}$ Department of Pathology, Shanghai Tenth People's Hospital, Tongji University School of Medicine, Shanghai 200072, China \\ ${ }^{6}$ Department of Orthopedic Surgery, Zhongshan Hospital, Fudan University, Shanghai 200032, China \\ ${ }^{7}$ Shanghai Engineering Research Center of Molecular Therapeutics and New Drug Development, College of Chemistry and \\ Molecular Engineering, East China Normal University, Shanghai 200062, China \\ *These authors have contributed equally to this work \\ Correspondence to: Zhong-Wei Lv, email: shtjnmd@163.com \\ David A. Fu, email:061101021@fudan.edu.cn \\ Yu-Shui Ma, email: mayushui2015@126.com
}

Keywords: GBM, glioma, proteomics analysis, gene expression analysis, Bioinformatics Analysis

Received: May 24, $2017 \quad$ Accepted: August 26, $2017 \quad$ Published: October 19, 2017

Copyright: Song et al. This is an open-access article distributed under the terms of the Creative Commons Attribution License 3.0 (CC BY 3.0), which permits unrestricted use, distribution, and reproduction in any medium, provided the original author and source are credited.

\section{ABSTRACT}

Glioblastoma multiforme (GBM), the most aggressive and lethal primary brain tumor, is characterized by very low life expectancy. Understanding the genomic and proteogenomic characteristics of GBM is essential for devising better therapeutic approaches. Here, we performed proteomic profiling of $8 \mathrm{GBM}$ and paired normal brain tissues. In parallel, comprehensive integrative genomic analysis of GBM was performed in silico using mRNA microarray and sequencing data. Two whole transcript expression profiling cohorts were used - a set of 3 normal brain tissues and 22 glioma tissue samples and a cohort of 5 normal brain tissues and $\mathbf{4 9}$ glioma tissue samples. A validation cohort included 529 GBM patients from The Cancer Genome Atlas datasets. We identified 36 molecules commonly changed at the level of the gene and protein, including up-regulated TGFBI and NES and down-regulated SNCA and HSPA12A. Single amino acid variant analysis identified 200 proteins with high mutation rates in GBM samples. We further identified 14 differentially expressed genes with high-level protein modification, among which NES and TNC showed differential expression at the protein level. Moreover, higher expression of NES and TNC mRNAs correlated with shorter overall survival, suggesting that these genes constitute potential biomarkers for GBM. 


\section{INTRODUCTION}

Glioma, which arises from glial cells, accounts for $80 \%$ of all malignant brain tumors [1]. The World Health Organization divides gliomas into four grades, of which glioblastoma multiforme (GBM) (grade IV) is the most aggressive and fatal. The median survival of GBM is approximately 15 months, even with standard-ofcare treatment [2]. Therefore, it is vital to illuminate the molecular basis of GBM to develop effective therapies.

Recently, next-generation sequencing assays have provided platforms for identifying cancer susceptibility genes [3]. The cancer genome atlas (TCGA) has performed an integrative analysis of DNA copy number, gene expression and DNA methylation aberrations in GBMs [4]. However, the mRNA levels do not always correlate with the protein abundance, and the major proteome of GBM has not been fully elucidated $[5,6]$. Thus, an integrated analysis of genomic and proteomic data may provide a more comprehensive understanding of the information flow that determines the GBM phenotype.

In this study, we performed label-free quantitative proteomics analysis for proteomic profiling and comprehensive integrative genomic analysis based on GEO and TCGA datasets of GBM to identify commonly changed molecular at the level of the gene and protein. Single amino acid variant analysis was also applied to identify proteins with high mutation rates in GBM samples. Our integrated analysis of the genomics and proteomics of GBM may provide a molecular basis and valuable insight for a new improved therapy of this disease.

\section{RESULTS}

To systematically screen the mRNA levels of dysregulated genes in glioma development, we analyzed an Affymetrix U133 plus 2.0 microarray expression database containing 3 normal brain tissues and 22 glioma tissue samples, including 15 low grade glioma (grade I-II) and 7 GBM samples (GEO dataset: GSE45921). For low grade glioma compared to normal brain tissues, 492 genes displayed $\geq 2$-fold difference at the $P<0.05$ level and 45 genes displayed $\geq 5$-fold difference at the $P<0.01$ level (Supplementary Figure 1A, 1B) for GBM compared to normal brain tissues, 657 genes displayed $\geq 1.5$-fold difference at the $P<0.05$ level and 20 genes displayed $\geq 5$-fold difference at the $P<0.01$ level (Supplementary Figure 1C, 1D).

For verification, we also analyzed an BiostarH-140s $\times 32$ microarray gene expression database with 3 normal brain tissues and 49 glioma tissue samples, including 22 low grade glioma and 27 GBM samples (GEO dataset: GSE51146). For low grade glioma compared to normal brain tissues, 399 genes displayed $\geq 2$-fold difference at the
$P<0.05$ level and 21 genes displayed $\geq 5$-fold difference in expression at the $P<0.01$ level (Supplementary Figure 2A, 2B) for GBM compared to normal brain tissues, 609 genes displayed $\geq 1.5$-fold difference in expression at the $P<0.05$ level and 25 genes in $27 \mathrm{GBM}$ tissue displayed $\geq 5$-fold difference at the $P<0.01$ level (Supplementary Figure 2C, 2D). Among the 609 genes in the GSE51146 dataset and the 657 genes in the GSE45921 dataset that displayed $\geq 1.5$-fold difference in expression in GBM and normal brain tissue, there were 148 common differentially expressed genes.

To examine stage-specific expression, we analyzed the differences between genes expressed in low-grade glioma and GBM tissues. For the GSE45921 database, 269 genes displayed $\geq 2$-fold difference at the $P<0.05$ level and 12 genes displayed $\geq 5$-fold difference at the $P<0.01$ level (Figure 1A, 1B). For the GSE51146 database, 136 genes in 27 GBM tissue displayed $\geq 2$-fold difference at the $P<0.05$ level and 23 genes displayed $\geq$ 5 -fold difference at the $P<0.01$ level (Figure 1C, 1D). Furthermore, there was little overlap in the genes that were expressed in low-grade glioma versus normal brain tissue and in GBM versus normal brain tissue in each of the data sets, suggesting that completely different biological processes mediate the development of low-grade glioma and GBM.

Next, we assessed Gene Ontology (GO) (Supplementary Figure 3A-3C), Kyoto Encyclopedia of Genes and Genomes (KEGG) pathways (Supplementary Figure 3D) and protein-protein interactions using STRING online analysis. Some functionally important proteins encoded by the 148 genes were identified, including polo like kinase 4 (PLK4), a tumor suppressor in GBM [7]; cyclin dependent kinase 6 (CDK6) [8]; PR/SET domain 10 (PRDM10) and myocyte enhancer factor 2C (MEF2C) (Supplementary Figure 3E).

Next, to further assess specific dysregulated protein in the occurrence and development of glioma, we performed LC-MS/MS-based whole proteomic profiling of $8 \mathrm{GBM}$ and paired adjacent normal brain tissues in triplicate. In all, 693 proteins displayed $\geq 1.5$-fold difference at the $P<0.05$ level (Supplementary Figure $4 \mathrm{~A})$, and 15 significantly dysregulated proteins displayed $\geq 10$-fold change at the $P<0.01$ level (Supplementary Figure 4B). We performed GO (Supplementary Figure 4C-4E), KEGG pathway and protein-protein interaction analysis on the 693 differentially expressed proteins using STRING analysis. The top 5 KEGG pathways were oxidative phosphorylation, Huntington's disease, Parkinson's disease, Alzheimer's disease, and metabolic pathways (Supplementary Figure 4F).

To further evaluate whether the differential expression of the 693 proteins identified by LC-MS/MS proteins could be observed at the transcript level, we assessed overlap with mRNA expression. Sixteen of the 693 proteins were 
in common with the 148 GBM-specific genes identified by gene microarray analysis, (Figure $2 \mathrm{~A}$ ). We verified the consistent expression trend of the most significantly upregulated (nestin [NES] and hexosaminidase subunit beta [HEXB]) and the most significantly down-regulated (heat shock protein family A member 12A [HSPA 12A], myelin basic protein [MBP]) genes/proteins by IHC (Figure 2B) and qRT-PCR (Figure 2C, 2D).

We also downloaded a GBM gene sequencing dataset (IlluminaHiSeq) from TCGA on the UCSC Genome Browser, which included 167 GBM and 5 normal brain tissue samples. There are 2881 genes differentially expressed in the GBM tissues (Fold change $\geq 1.5 ; P<$ 0.05 ) (Figure $3 \mathrm{~A}$ ), among which only 10 were in common with the 693 differentially expressed proteins identified in our study (Figure 3B).

In another GBM gene microarray dataset with 529 GBM and 10 normal brain tissue samples, 298 genes were differentially expressed in GBM tissues (Fold change $\geq$
$1.5 ; P<0.05$ ) (Figure 3C), with only 14 genes in common with our set of 693 differentially expressed proteins (Figure 3D).

Integrated analysis of the 693 GBM-specific proteins with the GBM-specific genes from 3 cohorts revealed a total of 36 differentially expressed genes/ proteins (25 up-regulated and 11 down-regulated) (Figure 3E). Overlapping molecules in two groups included up-regulated transforming growth factor beta induced (TGFBI) [9] and Nestin (NES) [10]; and downregulated synuclein alpha (SNCA) [11] and heat shock protein family A member 12A (HSPA12A), which have demonstrated roles in GBM and/or other cancers. These results suggest that the integrated analysis was effective in identifying potential molecular markers of GBM.

Furthermore, we performed GO (Supplementary Figure 5A-5C), KEGG pathway and protein-protein interaction analysis on the 36 cross-regulated genes/ proteins. The top $5 \mathrm{KEGG}$ pathways were Parkinson's

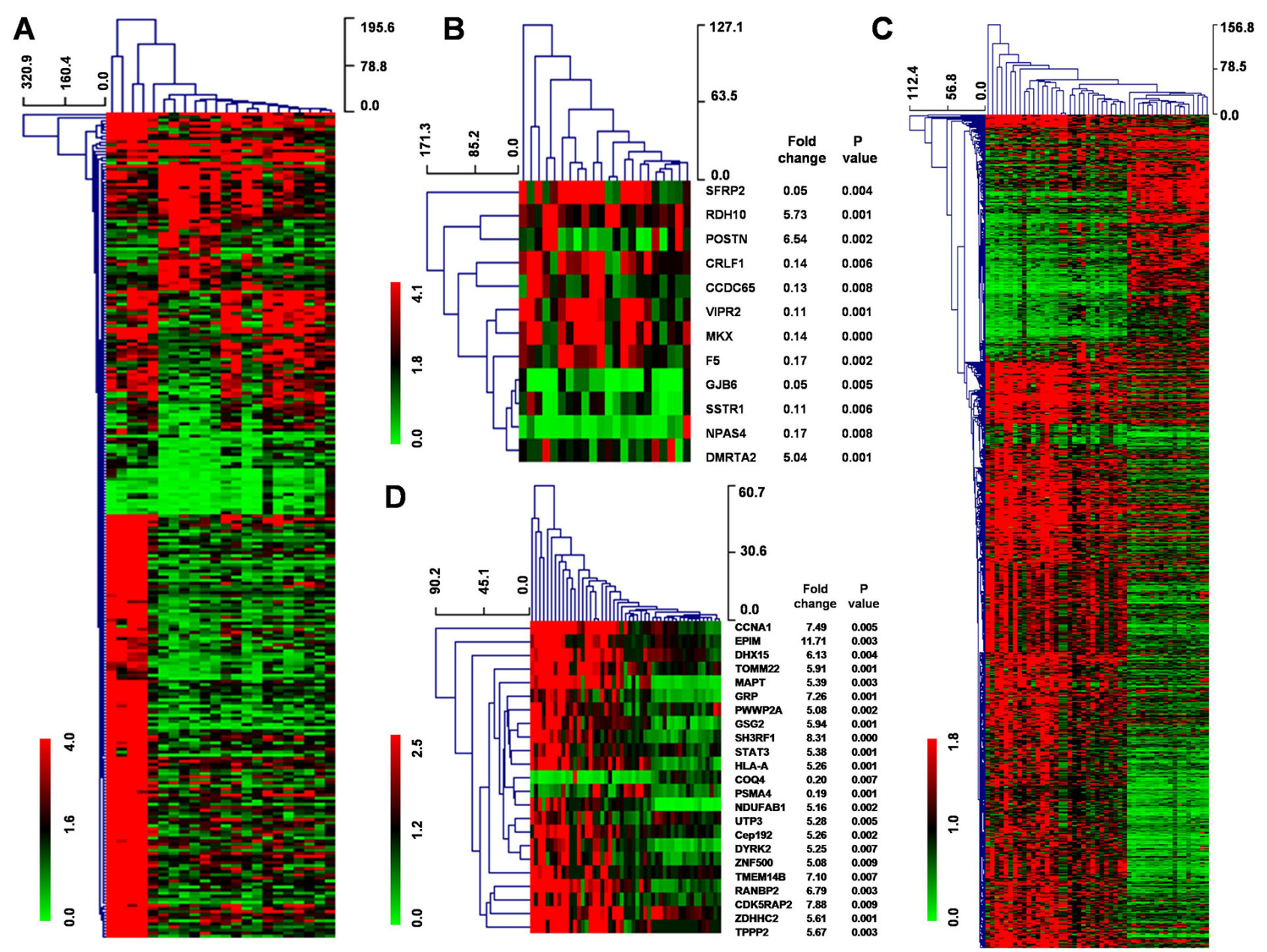

Figure 1: Hierarchal clustering of genes that are differentially expressed in low-grade glioma and GBM tissue. (A) Hierarchical clustering for 269 differentially expressed genes (Fold change $\geq 2 ; P<0.05$ ) in 7 GBM tissues compared to 15 low-grade tissues using MEV4.7.1 software. (B) Hierarchical clustering for 12 differentially expressed genes (Fold change $\geq 5 ; P<0.01$ ) in 7 GBM tissues compared to 15 low-grade tissues using MEV4.7.1 software. (C) Hierarchical clustering for 136 differentially expressed genes (Fold change $\geq 2 ; P<0.05$ ) in 27 GBM tissues compared to 22 low-grade tissues using MEV4.7.1 software. (D) Hierarchical clustering for 23 differentially expressed genes (Fold change $\geq 5 ; P<0.01$ ) in 27 GBM tissues compared to 22 low-grade tissues using MEV4.7.1 software. 
disease, metabolic pathways, retrograde endocannabinoid signaling, oxidative phosphorylation, and neuroactive ligand-receptor interaction (Supplementary Figure 5D). Protein-protein interaction networks identified signal transducer and activator of transcription-3 (STAT3), ELAV like RNA binding protein 1 (ELAVL1), CD44 and calmodulin 1 (CALM1) (Supplementary Figure 5E).

As variant peptides are not included in the standard UniProt Human database (36858 peptides in 4834 proteins), we created a customized mutation database (23405 mutated peptides in 2515 proteins; mean 9.3 mutated peptides per protein) to identify single amino acid variants (SAAVs). Our results revealed 3884 peptide mutation in 897 proteins (mean 4.3 mutated peptides per protein) (Figure 4A). Fourteen peptides of 14 proteins were mutated only in 1 of 8 normal brain tissues (mutation rate $12.5 \%$ ) but in $\geq 6$ of $8 \mathrm{GBM}$ tissues (mutation rate $75 \%$ ) (Figure 4B). Additionally, 335 peptides of 186 proteins were mutated exclusively in GBM tissue (mutation rate $100 \%)$, of which, $150(80.6 \%)$ had only a single SAAV, $20(10.8 \%)$ had 2 SAAVs, $13(7.0 \%)$ had 2-10 SAAVs and
$3(1.6 \%)$ had more than 10 SAAVs (MYH11, 48 SAAVs; FN1, 16 SAAVs; SYNM, 14 SAAVs) (Figure 4C-4D).

KEGG pathway enrichment analysis for the 200 proteins with $\geq 75 \%$ mutation rate indicated that 19 proteins $(9.5 \%)$ were enriched in the "Focal adhesion pathway" (Figure 5A). Additionally, 6 variant proteins were represented within the 148 differentially expressed genes from microarray, 3 were represented within the 298 differentially expressed genes from TCGA array, and 8 were represented within the 2881 differentially expressed genes from TCGA sequencing (Figure 5B). Two of the 14 molecules (NES and TNC) also showed differential protein expression in the LS-MS/MS assay.

We analyzed the clinical significance of NES and TNC using data of 529 GBM patients from TCGA datasets and $27 \mathrm{GBM}$ patients from our gene expression array. High expression of NES (Figure 5C, 5D) and TNC (Figure $5 \mathrm{E}, 5 \mathrm{~F}$ ) correlated with shorter overall survival, suggesting that these two molecules are potential biomarkers for GBM, suggesting that these two molecules constitute potential biomarkers for GBM.
A

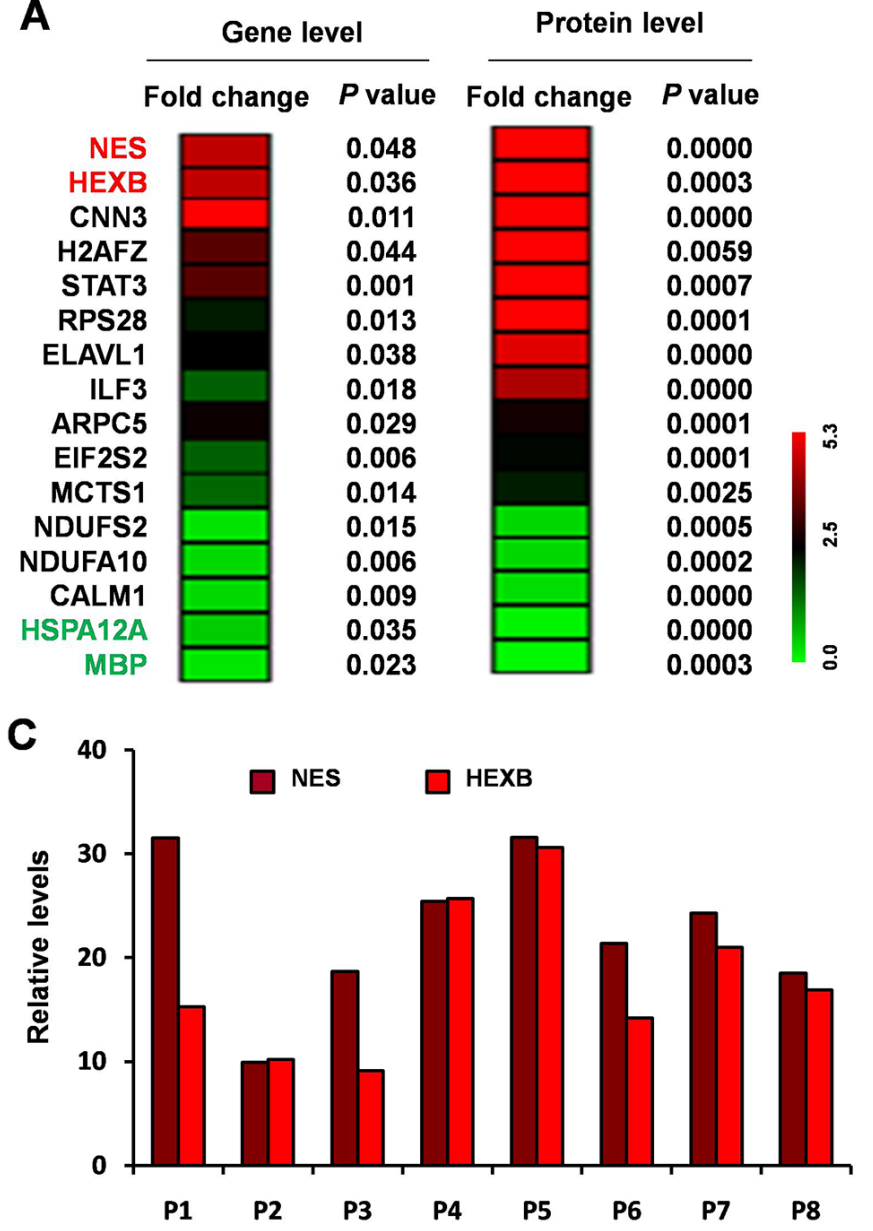

B
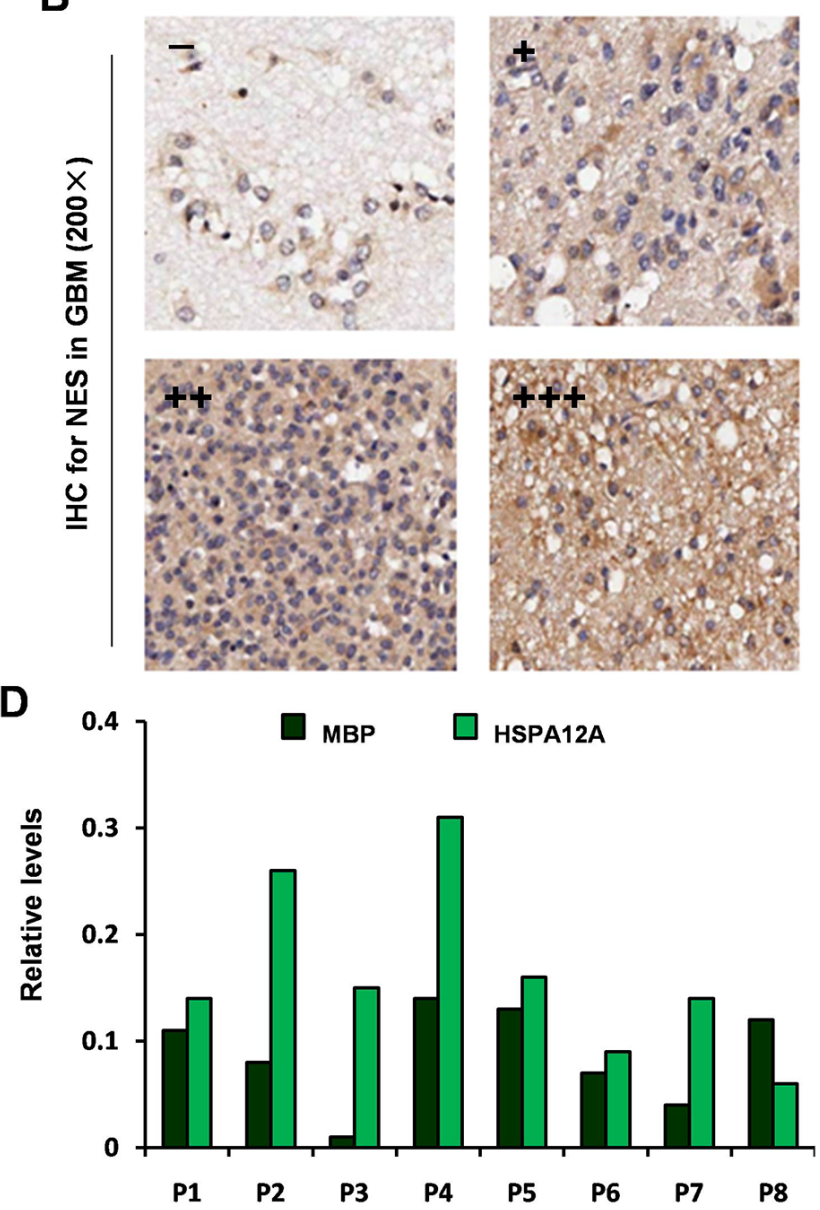

Figure 2: Integration of the differentially expressed proteins and genes from microarray. (A) Heat maps for 16 common genes among the 148 differentially expressed genes that had similar expression patterns as corresponding proteins among the 693 differentially expressed proteins. (B) NES antibody staining of GBM tissues. (C) Relative expression level of NES and HEXB by qRTPCR. (D) Relative expression level of HSPA12A and MBP by qRT-PCR. 


\section{DISCUSSION}

GBM is the most aggressive and lethal primary brain tumors, characterized by very low life expectancy. In this study, we present an integrated study of genomic and proteomics in GBM and paired normal brain tissues, which provides a number of insights into the biology of GBM and identifies potential therapeutic targets.

Through gene expression analysis based on GEO datasets and TCGA datasets and proteomic analysis, we identified 36 molecules commonly changed at the level of the gene and protein. Our results revealed that mRNA transcript abundance could relatively reliably predict protein abundance differences. Interestingly, we found that many transcripts are expressed differentially between the low-grade glioma and GBM tissue samples. Our results also suggest that distinct biological process may characterize different stages of glioma.

In this study, several of the differentially expressed molecules identified, including TGFBI, NES, SNCA, and HSPA12A, have demonstrated roles in GBM and other cancers. TGFBI encodes an RGD-containing protein that binds type I, II and IV collagens and is functionally upregulated in GBM upon TGF-beta signaling [9]. NES, which encodes a member of the intermediate filament protein family, is expressed primarily in nerve cells and is a prognostic marker in GBM [10]. Alpha-synuclein is a member of the synuclein family, which is abundantly expressed in the brain; its overexpression increased the vulnerability of U373 cells to apoptosis through TNFalpha-mediated pathways [11]. Furthermore, our result of proteins and pathways identified by GO and KEGG, including STAT3and CD44, were in agreement with that of previous reports, which verified their function in GBM. STAT3 has been clearly implicated in the development, progression, and aggressiveness of GBM [12]. CD44, a transmembrane molecule with several isoforms, is overexpressed in many tumors, including GBM and has been implicated in malignant processes including cell motility, tumor growth, and angiogenesis [13]. Until now, clinical trials investigating CD44 targeting in CD44positive solid tumors are underway, and the evidence presented in the previous report suggests that CD44 inhibition in GBM may be a promising therapy.
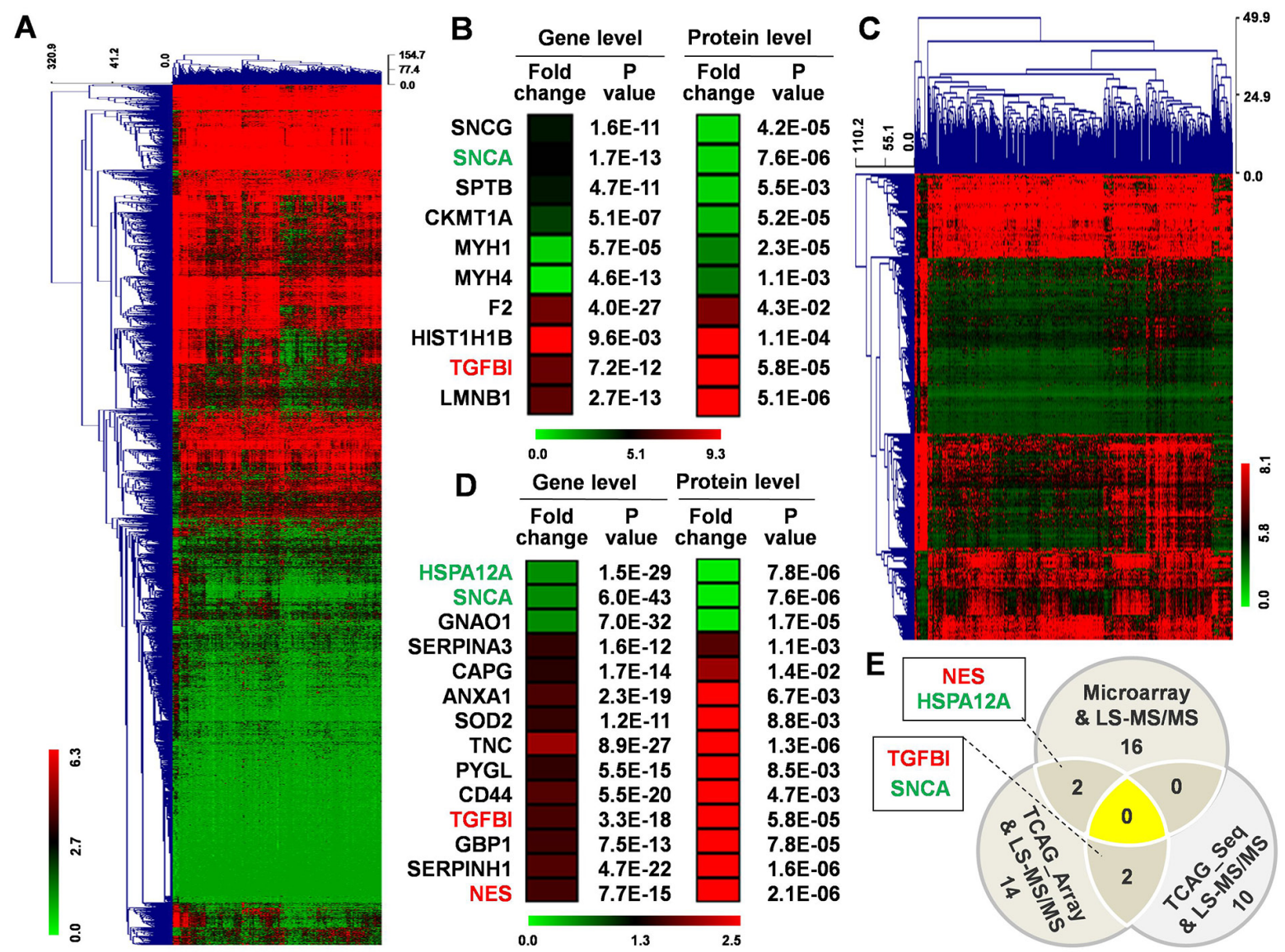

Figure 3: Analysis of TCGA datasets and comparison with protein expression data. (A) Hierarchical clustering for 2881 differentially expressed genes (Fold change $\geq 1.5 ; P<0.05$ ) in 167 GBM tissue compared to normal brain tissue using MEV4.7.1 software. (B) 10 common genes contained among the 2881 differentially expressed genes and the 693 differentially expressed proteins. (C) Hierarchical clustering for 298 differentially expressed genes (Fold change $\geq 1.5 ; P<0.05$ ) in 529 GBM tissues compared to normal brain tissue using MEV4.7.1 software. (D) 14 common genes contained among the 298 differentially expressed genes and the 693 differentially expressed proteins. (E) 36 changed genes/proteins among three groups. 



Figure 4: Single amino acid variants associated with GBM. (A) the numbers of proteins and peptides in the standard protein library (Standard) and our customized SAAV database of GBM (SAAVs); and the numbers of mutated proteins/peptides uniquely identified in this study (Mut). Standard database included 36858 peptides in 4834 proteins; mean 7.6 peptides per protein and our customized mutation database included 23405 mutated peptides in 2515 proteins; mean 9.3 mutated peptides per protein. We identified 3884 peptide mutations in 897 proteins; mean 4.3 mutated peptides per protein. (B) the number of samples with mutations in 14 peptides of 14 proteins in GBM and normal brain samples with $75-99 \%$ mutation rate. (C) the percent of proteins with different mutation numbers among those with SAAVs that were exclusively observed in GBM (100\% mutation rate). Blue, $1 \mathrm{SAAV}$; red, 2 SAAVs; green, 2-4 SAAVs; and purple, more than 4 SAAVs. (D) the number of mutation sites and cases of GBM for the 3 most frequently mutated proteins (MYH11, FN1 and SYNM). Red boxes indicate the mutation site and mutation cases in 8 GBM patients.

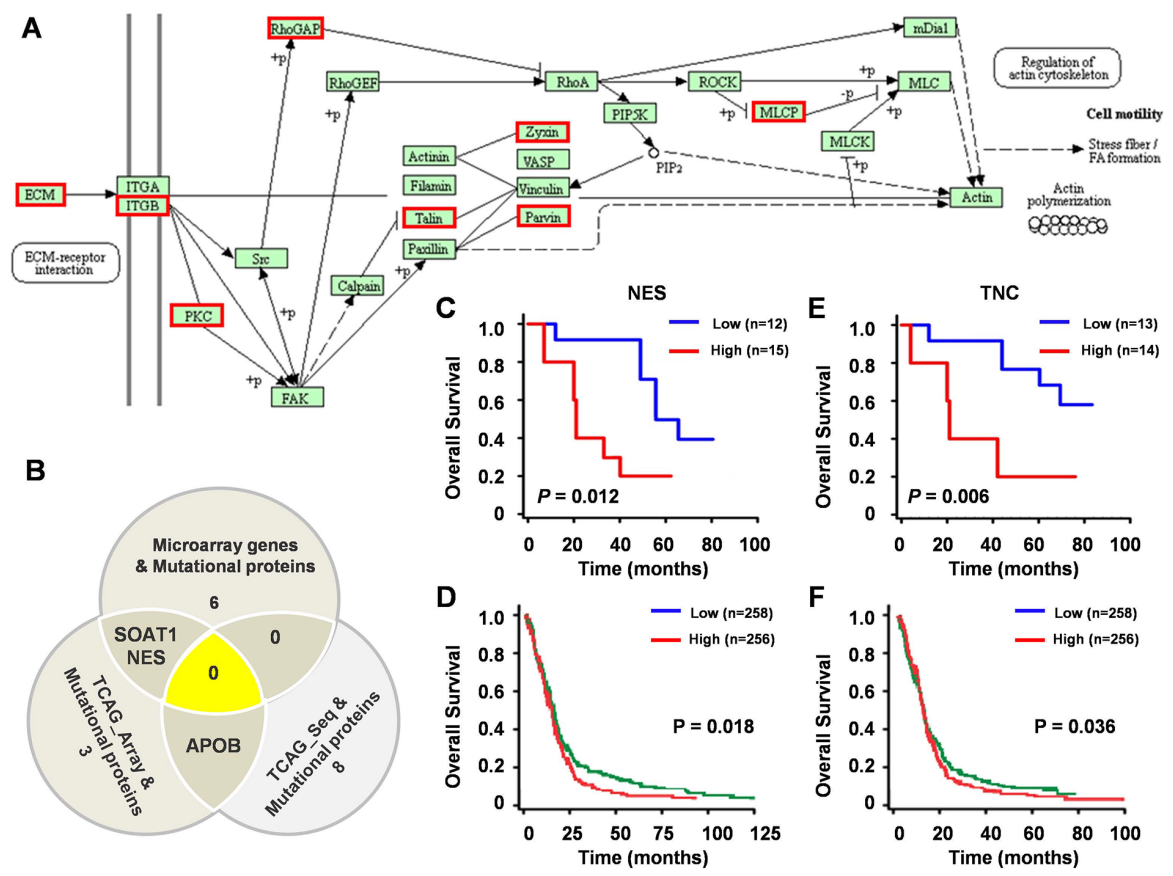

Figure 5: Clinical significant of mutated proteins. (A) 19 proteins (9.5\%) enriched in the Focal adhesion pathway by KEGG pathways classification enrichment analysis. Red boxes indicate the proteins in the Focal adhesion pathway that were mutated in GBM. (B) 14 commonly identified genes among three groups. SOAT1, NES and APOB were identified in more than one group. Univariate analysis of OS and expression of NES mRNA in 27 GBM patients (C) and 514 GBM patients (D) Univariate analysis of OS and expression of TNC mRNA in 27 GBM patients (E) and 514 GBM patients (F). 
A fundamental question in proteogenomics is to identify expressed protein coding alterations at the protein level. However, standard database search approaches cannot identify variant peptides from MS/MS data. We created a customized mutation database of GBM to performed database searches for SAAVs from the COSMIC database and performed database searches with customized sequence databases to identify SAAVs. Our SAAVs analysis identified 200 proteins with high mutation rates in GBM samples. To better understand the biology underlying the proteomic variants, we further identified 14 differentially expressed genes with high-level protein modification, among which NES and TNC showed differential expression at the protein level. Moreover, higher expression of NES and TNC mRNAs correlated with shorter overall survival, which suggesting that cancer-related variant proteins may serve as candidate protein biomarkers or therapeutic targets.

Taken together, our proteomic characterization of the genomically annotated GBM clarifies the power of integrated proteomics and genomics analysis and provides a molecular basis and valuable insight for new advances in GBM therapy. Moreover, our protein coding alteration approach provided new insights into their roles in GBM and may be broadly extended to understand roles of protein mutation in other cancers.

\section{MATERIALS AND METHODS}

\section{Tissue samples and ethics statement}

The study protocol and acquisition of tissue specimens were approved by the Ethical Committee of Shanghai Tenth People's Hospital, Tongji University School of Medicine, and Ganzhou City People's Hospital (2015-Res-10). This study was registered with ClinicalTrials.gov, number NCT01454102. Each participant provided written informed consent before participating in this study. We collected 8 GBM samples and paired normal brain tissue from patients undergoing surgical resection and classified according to the last WHO classification of central nervous tumors, which was confirmed by two experienced pathologists independently.

\section{RNA extraction}

According to the manufacturer's guideline, total RNA was isolated using Trizol regent (Invitrogen, Carlsbad, CA, USA). RNA quantity was determined using NanoDrop ND-1000 spectrophotometer and the integrity of RNA was measured by gel electrophoresis.

\section{Real-time quantitative reverse transcription PCR}

cDNA was synthesized from total RNA (10 ng), and quantitative PCR reactions were performed with the Taqman ${ }^{\mathrm{TM}}$ Universal PCR Kit (Life Technologies).
GAPDH was used as the internal control, and the $2^{-\Delta \Delta \mathrm{CT}}$ method was used to analyze the expression levels of genes.

\section{Immunocytochemistry}

GBM paraffin sections were cut into $4-\mu \mathrm{m}$ thick sections, then added onto poly-lysine coated slides and incubated at $65^{\circ} \mathrm{C}$ overnight. The incubated slides were then deparaffinized in xylene and rehydrated with graded alcohol. Next, retrieve the antigen using citrate buffer $(\mathrm{pH}$ 6.0) and store the slides in Tris buffered saline. In order to block endogenous peroxidase activity, 3\% hydrogen peroxide was added to the slides. They were then incubated overnight at $4^{\circ} \mathrm{C}$ in monoclonal antibody (Novus, Littleton, CO, USA) solution at 1:200 dilution. Finally, the slides were incubated with horseradish peroxidase-conjugated goat antirabbit immunoglobulin, and color was developed using the DAB Horseradish Pe-roxidase Color Development Kit (Maixin Co., Fuzhou, China).

\section{Protein extraction and analysis by nano-LC-MS/ MS}

Paired 8 GBM and normal brain tissues were cut into small pieces (about $1 \mathrm{~mm}^{3}$ ) and rinsed in PBS to remove blood. Then tissue were homogenized in $4 \%$ SDS and $0.1 \mathrm{M}$ DTT in $0.1 \mathrm{M}$ Tris- $\mathrm{HCl}, \mathrm{pH} 7.6$ solution on ice, sonicated 10 times ( $80 \mathrm{w}$, working $10 \mathrm{~s}$, suspending $15 \mathrm{~s}$ ), incubated for $5 \mathrm{~min}$ at $95{ }^{\circ} \mathrm{C}$. The protein concentrations of clarified lysates were determined using fluorescence assay. A $200 \mu \mathrm{g}$ of clarified lysates were proteolysedon 10 kDa Filter (PALL Life Sciences, USA) using a Filter Aided Sample Preparation (FASP) method. The peptide samples were then desalted onto a solid-phase extraction cartridge (Empore $7 \mathrm{~mm} / 3 \mathrm{ml}$ ). The lyophilized peptide mixture was re-suspended in water with $0.1 \%$ formic acid (v/v) and its content was estimated by UV light spectral density at 280 $\mathrm{nm}$, then $3 \mu \mathrm{g}$ of the digest peptides were analyzed by nanoliquid chromatography-tandem mass spectrometry (LC-MS/ MS) on LTQ Orbitrap Velos Pro mass spectrometer [14].

Raw data were processed by by Maxquant software (1.3) and then used for database and spectral library searching using Andromeda peptide search engines. The Maxquant peptide and protein quantification results files were imported into Perseus software (version 1.5.1.6) for further analysis. All of the MS proteomics data have been deposited to iProX (http://www.iprox.org/index) and can be accessed with the accession IPX00084901.

\section{Bioinformatic analysis}

The expression levels of genes were investigated in paired GBM tissue samples based on GEO datasets (GSE45921 and GSE51146) using the NCBI Platform (http://www.ncbi.nlm.nih.gov/) and TCGA datasets (TCGA-IlluminaHiSeq and TCGA_GBM_Exp_U133a) from UCSC Genome Browser (https://genome-cancer. 
ucsc.edu/). Hierarchical clustering was performed using the multiple experiment viewer $(\mathrm{MeV})$ 4.7.1 software (http://www.tm4.org/mev/).

We used GO classifications (http://www. geneontology.org/) to evaluate the biological function of the changed genes in GBM through three aspects including biological process, molecular function and cellular components. Subsequently, dysregulated genes were subjected to KEGG pathway analysis. Proteinprotein interaction networks analysis was performed by STRING (http://string-db.org/).

\section{Statistical analysis}

The results were expressed as mean \pm S.D. (standard deviation). Statistical significance between the groups was assessed by using one-way analysis of variance (ANOVA). Univariate survival analysis and multivariate analyses were carried out using the Kaplan-Meier method. All calculations were performed with the SPSS 20.0 software program (SPSS Inc, Chicago, IL, USA). The level of significance was chosen as $P<0.05$.

\section{Abbreviations}

GBM: Glioblastoma multiforme; GO: Gene Ontology; KEGG: Kyoto Encyclopedia of Genes and Genomes; LC-MS/MS: liquid chromatography-tandem mass spectrometry; SAAVs: single amino acid variants; TCGA: The cancer genome atlas.

\section{Author contributions}

YCS, GXL, HWZ, XMZ, XLC, SBX, RK, ZWL, DAF and YSM designed the study. YCS, GXL, HWZ, XMZ, XLC, SBX, RK, ZYC, RS, LC, RTX, MXC, MS, WQM, HQY, SYF, TMW, QX, ZWL, DAF and YSM collected and analyzed the sample. YCS, GXL, HWZ, SBX, RK, DL, ZYC, XFW, YJZ, LC, RTX, MXC, MS, WQM, HQY, YCSh, SYF, TMW, QX, DAF and YSM analyzed the genetic data. YCS, GXL, HWZ, SBX, RK, DL, ZYC, RS, LC, RTX, MXC, WQM, HQY, SYF, TMW, $\mathrm{QX}, \mathrm{ZWL}, \mathrm{DAF}$ and YSM performed the proteogenomic experiments and analyzed the data. YCS, GXL, HWZ, $\mathrm{XMZ}, \mathrm{XLC}, \mathrm{SBX}$ and RK contributed equally to this work. All authors contributed to the final version of the manuscript.

\section{ACKNOWLEDGMENTS}

We thank all study subjects for their participation. We appreciate experimental support of Central Laboratory for Medical Research, Shanghai Tenth People's Hospital. We thank LetPub (www.letpub.com) for its linguistic assistance during the preparation of this manuscript.

\section{CONFLICTS OF INTEREST}

The authors declare no potential conflicts of interest.

\section{GRANT SUPPORT}

This study was supported partly by grants from the National Natural Science Foundation of China (81201535, 81472202, 81772932, 81472209 and 81302065), Jilin Provincial Science and Technology Department (20140414061GH), Shanghai Natural Science Foundation (12ZR1436000 and 16ZR1428900) and Shanghai Municipal Commission of Health and Family Planning (201440398 and 201540228).

\section{REFERENCES}

1. Wang HX, Xu T, Jiang Y, Xu HC, Yan Y, Fu D, Chen JX. The challenges and the promise of molecular targeted therapy in malignant gliomas. Neoplasia. 2015; 17:239-255.

2. Brown TJ, Brennan MC, Li M, Church EW, Brandmeir NJ, Rakszawski KL, Patel AS, Rizk EB, Suki D, Sawaya R, Glantz M. Association of the extent of resection with survival in Glioblastoma: a systematic review and Metaanalysis. JAMA Oncol. 2016; 2:1460-1469.

3. Metzker ML. Sequencing technologies - the next generation. Nat Rev Genet. 2010; 11:31-46.

4. Cancer Genome Atlas Research Network. Comprehensive genomic characterization defines human glioblastoma genes and core pathways. Nature. 2008; 455:1061-1068.

5. Neilson KA, Ali NA, Muralidharan S, Mirzaei M, Mariani M, Assadourian G, Lee A, van Sluyter SC, Haynes PA. Less label, more free: approaches in label-free quantitative mass spectrometry. Proteomics. 2011; 11:535-553.

6. Dolgin E. Venerable brain-cancer cell line faces identity crisis. Nature. 2016; 537:149-50.

7. de Cárcer G, Escobar B, Higuero AM, García L, Ansón A, Pérez G, Mollejo M, Manning G, Meléndez B, AbadRodríguez J, Malumbres M. Plk5, a polo box domain-only protein with specific roles in neuron differentiation and glioblastoma suppression. Mol Cell Biol. 2011; 31:1225-39.

8. Bellail AC, Olson JJ, Hao C. SUMO1 modification stabilizes CDK6 protein and drives the cell cycle and glioblastoma progression. Nat Commun. 2014; 5: 4234.

9. Lin B, Madan A, Yoon JG, Fang X, Yan X, Kim TK, Hwang D, Hood L, Foltz G. Massively parallel signature sequencing and bioinformatics analysis identifies up-regulation of TGFBI and SOX4 in human glioblastoma. PLoS One. 2010;5:e10210.

10. Mangiola A, Lama G, Giannitelli C, De Bonis P, Anile C, Lauriola L, La Torre G, Sabatino G, Maira G, JhanwarUniyal M, Sica G. Nestin and c-Jun N-terminal kinase (JNK) expression in tumor and peritumor areas of 
glioblastoma multiforme: possible prognostic implications. Clin Cancer Res. 2007; 13:6970-6977.

11. Stefanova N, Schanda K, Klimaschewski L, Poewe W, Wenning GK, Reindl M. Tumor necrosis factor-alphainduced cell death in U373 cells overexpressing alphasynuclein. J Neurosci Res. 2003; 73:334-340.

12. Luwor RB, Stylli SS, Kaye AH. The role of Stat3 in glioblastoma multiforme. J Clin Neurosci. 2013; 20:907-11.
13. Mooney KL, Choy W, Sidhu S, Pelargos P, Bui TT, Voth B, Barnette N, Yang I. The role of CD44 in glioblastoma multiforme. J Clin Neurosci. 2016; 34:1-5.

14. Sun X, Zhang H, Luo L, Zhong K, Ma YS, Fan L, Fu D, Wan L. Comparative proteomic profiling identifies potential prognostic factors for human clear cell renal cell carcinoma. Oncol Rep. 2016; 36:3131-3138. 